\section{SEGREGACIÓN RESIDENCIAL SOCIOECONÓMICA E INSERCIÓN LABORAL: EL CASO DE SAN CARLOS DE BARILOCHE, ARGENTINA ${ }^{1}$}

\author{
Andrés Niembro², Tomás Guevara ${ }^{3}$ y Eugenia \\ Cavanagh $^{4}$
}

\section{Resumen}

La segregación residencial socioeconómica (SRS) puede considerarse uno de los principales obstáculos para lograr una adecuada integración urbana en las ciudades latinoamericanas, ya que suele entrelazarse con diversas problemáticas sociales y contribuir a su reproducción intergeneracional. El objetivo del artículo es realizar una investigación exploratoria sobre los vínculos entre la SRS y la inserción laboral de la población en el caso de la ciudad de San Carlos de Bariloche, Argentina. Mediante una combinación de técnicas de análisis multivariado y datos del último censo poblacional (2010), se obtiene primero una tipología que permite diferenciar a los barrios de la ciudad y, luego,

\section{SOCIOECONOMIC RESIDENTIAL SEGREGATION AND INCORPORATION INTO THE WORKPLACE: THE CASE OF SAN CARLOS DE BARILOCHE, ARGENTINA}

Andrés Niembro, Tomás Guevara y Eugenia Cavanagh

\section{Abstract}

Socioeconomic residential segregation (SRS) can be considered one of the main obstacles for an adequate urban integration in Latin American cities, since it is related to different social problems and contributes to their intergenerational reproduction. This article carries out an exploratory research on the links between SRS and the incorporation into the workplace of the population in San Carlos de Bariloche city, Argentina. Through a combination of multivariate analysis techniques and data from the last population census (2010), we obtain a typology that classifies the neighborhoods of the city and, then, we analyze the labor integration of the 
se analiza la integración al mercado de trabajo de la población que allí reside. Los resultados están en línea con trabajos previos de Latinoamérica, que muestran que quienes habitan en entornos segregados suelen tener mayores dificultades de inserción laboral y, con ello, de integración y progreso social. Por otro lado, parte de la evidencia no solo parece contradecir a las tradicionales tesis del desajuste espacial o del efecto vecindario, sino también mostrar algunos límites de las políticas públicas para incidir en la integración urbana plena si solo se limitan al acceso a la vivienda y servicios básicos.

\section{PALABRAS CLAVES: SEGREGACIÓN; CLASIFICACIÓN DE BARRIOS; INSERCIÓN LABORAL; DESEMPLEO; SAN CARLOS DE BARILOCHE.}

Recibido: 30-05-2019

Aceptado: 16-10-2019

1 Proyecto PICT 2015-3020, Agencia Nacional de Promoción Científica y Tecnológica; Universidad Nacional de Río Negro.

2 Argentina. Investigador Universidad Nacional de Río Negro, Centro Interdisciplinario de Estudios sobre Territorio, Economía y Sociedad (CIETES), http://orcid.org/0000-0003-1449-6361. Correo electrónico: aniembro@unrn.edu.ar. population located there. The results are in line with previous work from Latin America, which show that those who live in segregated environments tend to have greater difficulties in accessing the workplace and, with it, in achieving social integration and economic progress. On the other hand, part of the evidence not only seems to contradict the traditional thesis of spatial mismatch or neighborhood effect, but also shows some of the limits of public policies used to influence urban integration if they are only limited to give access to housing and basic services.

\section{KEYWORDS: SEGREGATION; NEIGHBORHOOD CLASSIFICATION; INCORPORATION INTO THE WORKPLACE; UNEMPLOYMENT; SAN CARLOS DE BARILOCHE.}

Received: 30-05-2019

Accepted: 16-10-2019

3 Argentina. Investigador Consejo Nacional de Investigaciones Científicas y Técnicas (CONICET); Universidad Nacional de Río Negro, Centro Interdisciplinario de Estudios sobre Territorio, Economía y Sociedad (CIETES) http://orcid.org/0000-0002-0535-4188. Correo electrónico: tguevara@ unrn.edu.ar

4 Argentina. Becaria doctoral Agencia Nacional de Promoción Científica y Tecnológica (ANPCYT). Universidad Nacional de Río Negro, Centro Interdisciplinario de Estudios sobre Territorio, Economía y Sociedad (CIETES), http://orcid.org/0000-0002-2042-1852. Correo electrónico: ecavanagh@unrn.edu.ar 


\section{Introducción}

El estudio sobre la segregación residencial socioeconómica (SRS) en las ciudades latinoamericanas se ha extendido rápidamente desde su emergencia a mediados de los años noventa. Después de décadas previas de discusión sobre desarrollismo, marginalidad y subdesarrollo, los estudios urbanos comenzaron a hacer hincapié, en el marco del Consenso de Washington y el pensamiento único, en las negativas consecuencias socioeconómicas de las transformaciones territoriales asociadas al neoliberalismo. Si bien el debate sobre la integración o exclusión urbana ha sido atravesado por diversos conceptos (dualización, polarización, segmentación, fragmentación, segregación, entre otros), todos reconocen, al margen de sus diferencias, el desarrollo de nuevos límites o fronteras urbanas al interior de las ciudades (Groisman y Suárez, 2010). Por otro lado, Brain, Cubillos y Sabatini (2007) afirman que la segregación ha mostrado ser un problema más crítico en América Latina de lo que se pensaba hasta hace algunos años, promoviendo una verdadera desintegración social de los barrios populares, con la consecuente expansión de problemáticas como el narcotráfico, la criminalidad, la deserción escolar o el desempleo crónico.

Esta investigación se enmarca en (e intenta ser un aporte a) la larga discusión sobre si la concentración espacial de la pobreza puede afectar o no las oportunidades de vida de los pobres urbanos, una pregunta que emerge del debate más amplio sobre las trampas de la pobreza y su transmisión o reproducción intergeneracional (Kaztman y Retamoso, 2005). Esto ha llevado a varios autores a analizar el vínculo entre la SRS y diversas problemáticas sociales, como la deserción escolar o la inactividad de los jóvenes, el embarazo adolescente, el desempleo o el tipo de inserción laboral de la población, entre otros (Cunha y Jakob, 2010; Groisman y Suárez, 2010; Kaztman, 1999; Kaztman y Retamoso, 2005; Perelman, 2011; Sabatini, Cáceres y Cerda, 2001; Sabatini y Wormald, 2013).

A diferencia de los estudios realizados en países desarrollados, donde la evidencia empírica sobre los efectos negativos de la concentración espacial de la pobreza es abundante, pero se tiende a indagar mucho menos en la integración de los individuos al mercado de trabajo (Korsu y Wenglenski, 2010), esta última dimensión es central en las investigaciones realizadas en ciudades latinoamericanas. En buena medida, esto se debe a que la inserción laboral y especialmente los ingresos laborales condicionan fuertemente el nivel de bienestar de los hogares más vulnerables (Perelman, 2011), dado el limitado o menor acceso y desarrollo relativo de los mercados de crédito y/o de las redes de protección social en América Latina (Groisman y Suárez, 2010). 
En este marco, donde la segregación residencial socioeconómica puede impactar en la inserción laboral de la población, pero también podría verse igualmente afectada por la inserción en el mercado de trabajo (ver sección "Efectos negativos de la SRS sobre la inserción laboral"), el objetivo del presente artículo es realizar una investigación exploratoria sobre los vínculos entre estas dimensiones en el caso de la ciudad de San Carlos de Bariloche (Argentina), a partir de datos del último censo poblacional (año 2010) y algunas técnicas de análisis multivariado (en línea con Dujardin, Selod y Thomas, 2008). Esta ciudad presenta algunas particularidades que la tornan un caso de estudio interesante. Ubicada en la Norpatagonia argentina, en el Oeste de la provincia de Río Negro, la ciudad está rodeada y atravesada por montañas, lagos, lagunas, ríos y arroyos (Figura 1), lo cual ya genera ciertas barreras para la integración urbana. A su vez, durante los últimos veinte años Bariloche ha experimentado un crecimiento poblacional sostenido, convirtiéndose en la ciudad más poblada de la provincia de Río Negro y en la tercera de la Patagonia argentina (luego de Neuquén y Comodoro
Rivadavia), lo que ha ido de la mano también de un importante y rápido proceso de expansión de su mancha urbana (Matossian, 2015). No obstante, se trata de una forma de urbanización más bien difusa y fragmentada, con bajas densidades de ocupación en grandes porciones del ejido municipal, el cual es a su vez uno de los más extensos del país (más de 27.000 hectáreas). Por otra parte, la preocupación por los problemas de desempleo o baja empleabilidad entre la población de los barrios populares, como así también por los vaivenes estacionales de la actividad turística y la construcción asociada, han sido frecuentes (Bosch, Suárez y Olivares, 2004; Niembro, 2011).

Luego de esta introducción, se repasa el marco teórico utilizado y algunos de los principales antecedentes. En la tercera, se presenta la metodología y las fuentes de información. En la cuarta, se analizan los resultados alcanzados, desde la definición de una tipología barrial para dar cuenta de la SRS en Bariloche hasta el análisis del vínculo con la inserción laboral de la población que habita dichos barrios. Por último, se presentan las reflexiones finales de este caso de estudio. 
FIGURA 1. MAPA SATELITAL DE BARILOCHE Y SU GEOGRAFÍA.

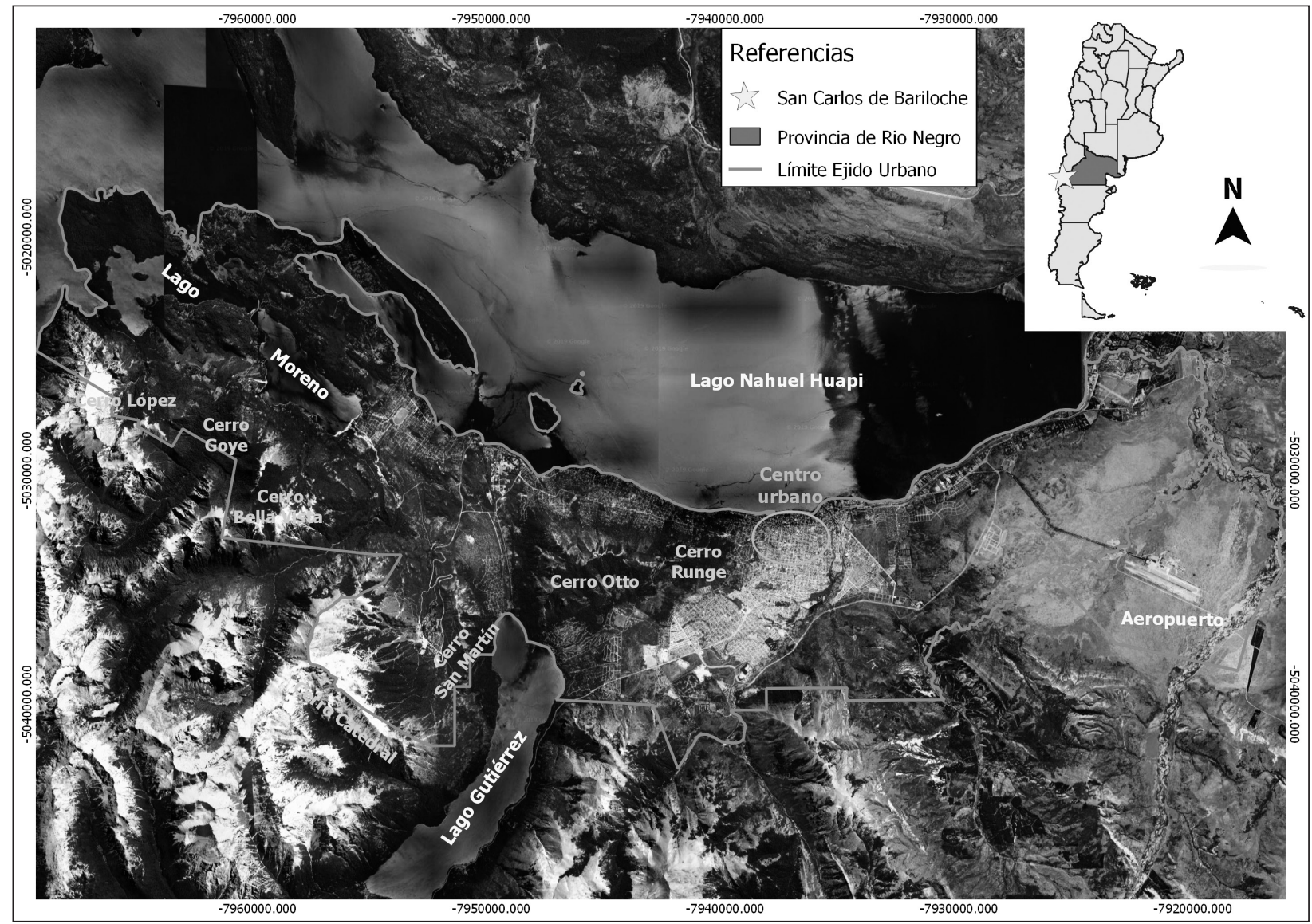

Fuente: elaboración propia en base a imagen Bariloche Google Satelital 2019 - Capa ejido urbano MSCB - SRC: EPSG:3857 - WGS 84 / Pseudo Mercator - Proyectado. 


\section{Marco teórico y antecedentes}

\section{INTEGRACIÓN URBANA Y SEGREGACIÓN RESIDENCIAL SOCIOECONÓMICA}

La integración urbana es uno de los principios y compromisos asumidos en la Nueva Agenda Urbana resultante de la Conferencia Hábitat III de 2016. Se propone como un atributo esencial de una ciudad sustentable y es un concepto multidimensional, que abarca no solo el acceso al suelo, vivienda e infraestructura de servicios, sino también a la dimensión laboral, cultural y política, entre muchas otras. De ahí la complejidad de su estudio y los debates sobre su posibilidad de medición y el tipo de políticas públicas necesarias para su abordaje.

Uno de los principales desafíos para la integración urbana tiene que ver con romper los obstáculos y los efectos negativos que tiene la SRS. Si bien un territorio segregado es, por definición, un territorio que presenta problemas de integración urbana, consideramos que no pueden reducirse como sinónimos. La integración urbana es un concepto más denso y abarcador que incluye diferentes dimensiones (Ruiz-Tagle, 2016). Por su parte, y aunque están intimamente vinculados, la SRS es un concepto más acotado, por lo que su operacionalización y abordaje en un trabajo de investigación resulta relativamente más simple, sin por ello estar exento de debates y discusiones (Rodríguez Merkel, 2013).
La SRS remite fundamentalmente a los patrones de localización de los diferentes grupos sociales en el espacio. Existen numerosos trabajos que, en función de diferentes variables socioeconómicas, demográficas y/o étnicas, buscan dar cuenta de las características de los barrios y de sus problemáticas sociales (Aguilar y Mateos, 2011; Mikelbank, 2011; Owens, 2012; Vicino, Hanlon y Short, 2011). Esta línea de investigación puede rastrearse desde los inicios mismos de los estudios urbanos, a fines del siglo XIX, y especialmente a principios del siglo XX con la Escuela de Chicago. En América Latina, la discusión sobre la segregación se instaló más fuertemente recién a partir de la década de 1990, al calor de las transformaciones urbanas derivadas de las políticas neoliberales del Consenso de Washington. Autores como Telles (1995), Kaztman (1999) y Sabatini et ál. (2001) pusieron de manifiesto las desventajas que implicaban los ambientes segregados de muchas ciudades latinoamericanas en términos de reproducción intergeneracional de la pobreza. En la actualidad, las investigaciones sobre SRS han cobrado auge en la región ante la evidencia de que la inequidad y la exclusión pueden aumentar con independencia de los procesos de reducción de la pobreza (Arriagada Luco, 2010). La SRS constituye así el cemento sobre el que las diferencias se asientan, reproducen y agravan, condicionando y dificultando posibles intentos de repliegue hacia situaciones de mayor igualdad y equidad social (Kaztman, 1999; Rodríguez Vignoli, 2008). 
Según Groisman y Suárez (2010, p. 8), la SRS se produce "en la medida en que las personas que comparten un mismo espacio urbano tienen también en común características socioeconómicas que las distinguen de otros grupos sociales que habitan otras áreas". Esta es una definición similar a la de Sabatini et ál. (2001), para quienes la SRS es la aglomeración geográfica de familias de una misma condición o categoría social. A su vez, estos autores reconocen tres dimensiones principales del fenómeno: 1) la tendencia de un grupo definido por características socioeconómicas similares, a concentrarse en algunas áreas; 2) la conformación de áreas socialmente homogéneas; y 3) la percepción subjetiva que tiene la gente de las dimensiones objetivas.

Entre las dimensiones señaladas, la conformación de áreas socialmente homogéneas es la que se vincula más fuertemente con el problema de desintegración social, ya que la homogeneidad social del espacio tiende a aislar a un grupo de los otros, lo cual es aún más grave si hablamos de grupos vulnerables (Molinatti, 2013a, 2013b). Precisamente, el aislamiento socioespacial de la población pobre hace que los mismos vean reducidos sus horizontes de posibilidades y sus probabilidades de acceder a una movilidad social ascendente (Kaztman, 1999). La SRS actúa así como un mecanismo (acumulativo o multiplicador) de reproducción de las desigualdades socioeconómicas, del cual ellas mismas son una manifestación (Rodríguez Vignoli y Arriagada Luco, 2004). Por ello, será principalmente este criterio de homogeneidad (y heterogeneidad) socio-residencial el que guiará en términos prácticos nuestro abordaje sobre la SRS. No obstante, reconocemos que el no poder incluir la dimensión subjetiva sobre las percepciones de los vecinos puede tomarse como una limitación en nuestro análisis, al igual que en otros tantos de la literatura empírica, lo cual constituiría un aporte enriquecedor a desarrollar en trabajos futuros.

\section{EFECTOS NEGATIVOS DE LA SRS SOBRE LA INSERCIÓN LABORAL}

Entre los enfoques teóricos más frecuentes sobre las consecuencias negativas de la SRS, podemos destacar dos: la teoría del efecto vecindario o barrio (neighbourhood effect); y la teoría de la geografía de oportunidades. La primera plantea que las acciones y posibilidades de los individuos están condicionadas directa e indirectamente por las características de su entorno barrial (Slater, 2013). Por ejemplo, cuando se piensa particularmente en los jóvenes, la exposición temprana o durante años formativos a ambientes segregados puede afectar negativamente sus trayectorias de vida y reducir así sus posibilidades de ascenso social. Esta experiencia barrial se puede traspasar o contagiar a través de diferentes mecanismos o redes sociales locales, como el efecto de pares, la socialización colectiva e institucional. 
Por su parte, la geografía de oportunidades plantea que existen variaciones tanto objetivas como subjetivas asociadas al proceso de toma de decisiones y las restricciones que el espacio plantea. La estructura, calidad y acceso a oportunidades varía de un barrio a otro. Al mismo tiempo, los valores, aspiraciones, preferencias y percepciones subjetivas acerca de los potenciales resultados de estas decisiones están afectados por la red social local y por la información que circula en el barrio y, por lo tanto, también varían geográficamente. Un corolario de esta perspectiva es lo que se denomina la tesis del desajuste espacial (spatial mismatch), según la cual las tasas diferenciales de desempleo entre los barrios se deben al desfasaje espacial entre los lugares de residencia de la mano de obra y las fuentes de empleo. En algunos casos, la distancia entre la oferta y la demanda puede implicar traslados más largos y costosos y desincentivar tanto la búsqueda o disposición laboral como la contratación -por ejemplo, si los empleadores discriminan a los trabajadores que tienen que desplazarse largos trayectos por considerarlos potencialmente menos productivos (Dujardin et ál., 2008). Asimismo, por el propio funcionamiento de las redes de contactos, pueden producirse asimetrías de información en la búsqueda de empleo que limiten el conocimiento sobre oportunidades laborales (Sabatini et ál., 2001).

Sea en un marco conceptual o en otro, la clave es que el espacio importa y que las características barriales (particularmente, los niveles de SRS) se vinculan con el grado y tipo de inserción laboral de los individuos (Cunha y Jakob, 2010; Kaztman, 1999; Molinatti, 2013a). No obstante, vale destacar que esta vinculación podría ser tranquilamente de doble vía (Dujardin et ál., 2008), es decir, la causalidad ir en un sentido o en otro. Por ello, Kaztman y Retamoso (2005) resaltan que cuando se analizan relaciones entre condiciones del contexto (como la segregación barrial) y características individuales (por ejemplo, la inserción laboral) es necesario tener una mirada crítica sobre la dirección de la causalidad. Esta bidireccionalidad puede representar, en todo caso, un proceso circular y vicioso, de reproducción intergeneracional de la pobreza. Si bien puede ocurrir que quienes por diferentes razones (muchas de ellas individuales) padecen los mayores problemas de inserción laboral deban (re)localizarse junto con sus familias en los barrios más pobres, es de esperar también, por las teorías antes reseñadas, que sus futuros vínculos con el mercado de trabajo suelan verse afectados por el contexto barrial (Molinatti, 2013a). En particular, los efectos de los vecindarios segregados podrían expresarse con mayor fuerza entre los jóvenes allí socializados, para los cuales no solo los excede la decisión de localización familiar, sino que en su caso la influencia de las características barriales antecede a sus decisiones y posibilidades de inserción laboral (Kaztman y Retamoso, 2005).

Por último, vale destacar que los intentos de analizar la causalidad en un único sentido, particularmente 
desde las características barriales hacia los resultados individuales, al estilo del efecto vecindario, han levantado varias críticas por caer en un determinismo espacial o en la reificación del espacio (Ruiz-Tagle y López, 2014; Slater, 2013). Por esto, y al igual que Cunha y Jakob (2010), creemos que sería temerario o imprudente querer desentrañar, si acaso es posible, el sentido de la causalidad entre estos fenómenos. Como se detallará en la siguiente sección, el objetivo de este trabajo es mucho más acotado y se limita a explorar la existencia o no de vínculos entre la SRS y la inserción laboral de la población de los diferentes barrios de Bariloche - una problemática que encuentra correlato en estudios previos sobre algunas grandes ciudades de Argentina (Groisman y Suárez, 2010; Molinatti, 2013a; Perelman, 2011; Salvia y Vergara, 2011).

\section{Metodología y datos}

A fin de analizar los vínculos entre la SRS y la inserción laboral en el caso de Bariloche, seguimos en buena medida el abordaje metodológico de: por un lado, Dujardin et ál. (2008), quienes en lugar de trabajar con índices (agregados o generales) de segregación desarrollan en primera instancia una tipología barrial, mediante técnicas de análisis multivariado, para así poder examinar espacialmente la SRS y las diferentes condiciones de los barrios; y por otro, de diversos autores latinoamericanos que, frente a ciertas limitaciones en los datos disponibles, realizan un análisis exploratorio de las relaciones entre la SRS y la inserción laboral de la población (Kaztman y Retamoso, 2005; Sabatini et ál., 2001; Sabatini y Wormald, 2013). Si bien debemos reconocer el esfuerzo reciente realizado por Grande (2016) para poder inferir datos a nivel individual a partir de los censos y del programa REDATAM (acrónimo de Recuperación de Datos para Áreas pequeñas por Microcomputador), también vale destacar que varios de los estudios que desarrollan ejercicios económétricos en base a microdatos sobre las características específicas de los individuos suelen recurrir, en primera instancia, a una evaluación exploratoria del vínculo entre SRS e inserción laboral (Cunha y Jakob, 2010; Dujardin et ál., 2008; Molinatti, 2013a; Perelman, 2011). Más importante aún es que las conclusiones a las que arriban tanto en el análisis exploratorio como en los modelos econométricos son coincidentes entre sí. En otros términos, incluso con la introducción de controles individuales (como edad, género, nivel educativo, entre otros), pruebas estadísticas y distintas estrategias para intentar resolver el problema de endogeneidad, los resultados siguen avalando la existencia de una relación entre el lugar de residencia (y la segregación barrial) y la vinculación de la población con el mercado de trabajo.

Dicho lo anterior, y como es usual en buena parte de la literatura empírica que estudia la SRS, nuestra fuente de datos es el último censo de población 
y vivienda (año 2010), cuya información se publica tradicionalmente al nivel de los diferentes radios censales en los que se divide el territorio (esto es, la unidad geoestadística más pequeña para la que se publican los resultados de los censos en Argentina). No obstante, para una mejor interpretación de la segregación residencial que experimentan los distintos grupos sociales resulta de una mayor riqueza analítica poder trabajar con las realidades barriales. Por ello, el primer paso ha sido barrializar el censo 2010 tomando los datos disponibles de los 159 radios censales que conforman el ejido de la ciudad de Bariloche. Mediante un trabajo cartográfico que consistió en revisar, superponer y contrastar las capas de barrios de Bariloche y de radios censales, se conformaron 77 unidades barriales.

Las variables que hemos recabado permiten cubrir varias dimensiones socioeconómicas y residenciales utilizadas tradicionalmente en la literatura especializada (Aguilar y Mateos, 2011; Marcos, Mera y Di Virgilio, 2015; Molinatti, 2013a, 2013b; Sánchez, Sassone y Matossian, 2007). Como puede apreciarse en la Tabla 1, las variables se organizan en diferentes ejes: necesidades básicas insatisfechas (NBI), infraestructura, régimen de tenencia, características de la vivienda, tenencia de bienes (proxy del poder adquisitivo) y educación del jefe de hogar. Para mayor claridad en la interpretación (lo veremos luego en la Tabla 2), se procuró expresar a las variables en un sentido positivo (es decir, ausencia de NBI, acceso a infraestructura, a bienes), salvo por el régimen de tenencia, donde se presentan tres tipos alternativos. En la Tabla 1 se aprecia, además, la existencia de importantes niveles de variabilidad en la mayoría de los indicadores, lo cual nos da un primer indicio de las heterogeneidades barriales en Bariloche (más adelante veremos que los dos valores extremos resaltados en negrita, relativos al régimen de propiedad, corresponden a un caso único).

Asimismo, el censo nos provee información sobre la condición laboral de la población, es decir, si se encuentran inactivos (por fuera del mercado de trabajo), ocupados o desempleados. A partir de estos datos absolutos podemos calcular y analizar las tasas de actividad, de empleo y de desocupación para las diferentes unidades barriales.

Los métodos de análisis multivariado adoptados en este trabajo se condicen con las técnicas generalmente utilizadas en esta literatura, en particular, la combinación de análisis de componentes principales (ACP) y análisis clúster (Dujardin et ál., 2008; Owens, 2012; Vicino et ál., 2011). En primer lugar, al analizar las variables incluidas en cada eje encontramos altos niveles de correlación dentro de los tres últimos (características de la vivienda, tenencia de bienes y educación del jefe de hogar), por lo que apelamos al ACP para obtener en cada caso un componente que condense la información o variabilidad compartida por estos indicadores (Hair, Black, Babin y Anderson, 2010; Johnson y Wichern, 2008). 
TABLA 1. VARIABLES UTILIZADAS OUE CARACTERIZAN A LOS HOGARES (EN PORCENTAJE DEL TOTAL DE HOGARES DEL BARRIO)

\begin{tabular}{|c|c|c|c|c|c|}
\hline & Media & $\begin{array}{l}\text { Desvío } \\
\text { estándar }\end{array}$ & $\begin{array}{l}\text { Coef. de } \\
\text { variac. }(\%)\end{array}$ & Mínimo & Máximo \\
\hline \multicolumn{6}{|l|}{ NBI } \\
\hline Sin ninguna necesidad básica insatisfecha & 90,1 & 11,5 & 12,8 & 46,7 & 100 \\
\hline \multicolumn{6}{|l|}{ Infraestructura } \\
\hline Acceso a gas de red & 85,6 & 20,2 & 23,6 & 21,2 & 100 \\
\hline Acceso a cloacas & 53,0 & 40,6 & 76,5 & 0 & 100 \\
\hline Acceso a red pública de agua & 89,7 & 21,2 & 23,6 & 12,4 & 100 \\
\hline \multicolumn{6}{|l|}{ Régimen de tenencia } \\
\hline Propietario (de la vivienda y el terreno) & 60,7 & 13,4 & 22,1 & 2,5 & 85,0 \\
\hline Inquilino & 20,0 & 13,4 & 66,8 & 0,5 & 55,0 \\
\hline Otros casos & 19,2 & 11,7 & 60,8 & 4,3 & 71,8 \\
\hline \multicolumn{6}{|l|}{ Características de la vivienda } \\
\hline Con baño dentro de la vivienda & 97,0 & 3,9 & 4,0 & 82,7 & 100 \\
\hline Con cañería de agua dentro de la vivienda & 94,6 & 8,0 & 8,4 & 59,2 & 100 \\
\hline Menos de 2 personas por cuarto & 84,7 & 12,0 & 14,2 & 40,8 & 98,8 \\
\hline \multicolumn{6}{|l|}{ Tenencia de bienes } \\
\hline Con heladera & 95,2 & 5,2 & 5,5 & 75,1 & 100 \\
\hline Con computadora & 62,4 & 17,4 & 27,9 & 11,4 & 92,0 \\
\hline Con teléfono fijo & 59,7 & 18,1 & 30,3 & 12,5 & 86,4 \\
\hline \multicolumn{6}{|l|}{ Educación del jefe de hogar } \\
\hline Completó la educación primaria & 88,4 & 9,6 & 10,9 & 59,8 & 100 \\
\hline Completó la educación secundaria & 51,3 & 24,6 & 47,9 & 7,1 & 89,8 \\
\hline Cursó y/o completó la educación superior & 30,6 & 22,0 & 72,0 & 1,6 & 73,2 \\
\hline
\end{tabular}

Fuente: elaboración propia en base al Censo 2010, Instituto Nacional de Estadísticas y Censos (INDEC). 
En segundo término, dado que el análisis clúster es sensible al uso de diferentes medidas, es necesario previamente estandarizar las variables, algo que se suele realizar por medio de $Z$ scores -i.e. a las variables originales se les resta la media y luego se dividen por el desvío estándar, de forma que una vez estandarizadas tengan media 0 y desvío 1 (Hair et ál., 2010; Johnson y Wichern, 2008). Por ello, convertimos en $Z$ scores los siete indicadores correspondientes a los ejes de NBI, infraestructura y régimen de tenencia, y agregamos a esta base los tres componentes principales derivados del paso anterior (vivienda, bienes y nivel educativo del jefe de hogar), que ya están estandarizados.

Finalmente, y en línea con el criterio de SRS adoptado, el análisis clúster busca maximizar la homogeneidad entre los casos incluidos dentro de cada conglomerado, al mismo tiempo que se maximiza la heterogeneidad entre los clústeres conformados. De las distintas alternativas para llevar a cabo este tipo de análisis, recurrimos a una de las técnicas jerárquicas comúnmente más empleada, el método de Ward, junto con la medida de proximidad recomendada, la distancia euclídea al cuadrado (Hair et ál., 2010; Johnson y Wichern, 2008). Vale destacar que esta misma técnica ha sido utilizada por varios de los trabajos especializados en la temática (Dujardin et ál., 2008; Marcos et ál., 2015; Mikelbank, 2011; Sánchez et ál., 2007). Para definir la cantidad final de clústeres seguimos el criterio (stopping rule) de evaluar el cambio porcentual en la heterogeneidad (la suma de los cuadrados de los errores intra-clúster que provee el método de Ward) para cada etapa del proceso aglomerativo, una de las reglas más simples y comúnmente utilizada entre las técnicas jerárquicas (Hair et ál., 2010).

\section{Resultados}

\section{SRS EN BARILOCHE: UNA TIPOLOGÍA BARRIAL}

En función de los criterios recién mencionados, optamos por una solución que agrupa a las 77 unidades barriales originales en 11 clústeres con marcadas y significativas diferencias entre sí (para más detalles técnicos sobre la determinación de la cantidad de clústeres, véase Niembro, Guevara y Cavanagh, 2019). Las características de estos tipos de barrios en Bariloche se pueden empezar a comprender a partir de la Tabla 2, el cual refleja el promedio de los diez indicadores para cada uno de los clústeres conformados. Por el proceso de estandarización, los valores responden la siguiente pregunta: ia cuántos desvíos estándar se encuentra el promedio grupal de la media general de todos los barrios de Bariloche, ya sea para arriba o para abajo? Dado que todas las variables socio-residenciales (desde NBI a Educación) están expresadas en términos positivos, los valores negativos dan cuenta precisamente de situaciones de 
carencia o vulnerabilidad: gris oscuro, cuando el promedio del clúster es menor a -1 ; intermedio, para valores entre -0,5 y -0,99; y claro, entre -0,49 y 0. Para las situaciones favorables, se resaltan los casos mayores a 1 (negrita) y los que se encuentran en el rango 0,5-0,99 (cursiva). Para homogeneizar el análisis, estos mismos criterios se aplican a las distintas variables que caracterizan al régimen de tenencia, pero allí no puede hacerse de antemano una valoración de casos favorables y desfavorables, sino que solamente ilustran la distancia entre el promedio del clúster y la media general.

En términos territoriales, el siguiente mapa (Figura 2) nos permite visualizar la localización de los barrios que conforman cada uno de los 11 clústeres y, a partir del cruce entre la Tabla 2 y el mapa, caracterizar a los distintos tipos de barrios en $\mathrm{Ba}-$ riloche (para detalles sobre los barrios incluidos en cada grupo, véase Niembro et ál., 2019). Un caso aparte es el clúster 7, el cual abarca a una sola unidad geográfica de análisis, conformada por el Barrio Militar y el Centro Atómico Bariloche, dos barrios o complejos habitacionales donde reside personal del Ejército Nacional y de la Comisión Nacional de Energía Atómica (CNEA). Por ende, la característica sobresaliente -más allá de poseer en promedio un alto nivel educativo y adquisitivo- es que son ocupantes por razones laborales de viviendas estatales pertenecientes a estos organismos públicos, las cuales cuentan con buenas condiciones habitacionales e infraestructura. Dadas estas características excepcionales y su tamaño acotado, este clúster se excluye del análisis siguiente.

\section{Barrios SUbURBanos POPULARES: 1 a 4}

Estos barrios se ubican mayormente en zonas suburbanas (o en los márgenes del centro urbano) hacia el Sur. Puntualmente, el clúster 1 abarca a barrios suburbanos de sectores bajos con restricciones en infraestructura. En general, son barrios de conformación reciente, con una cuota importante de ocupaciones informales que explica, en buena medida, su falta de consolidación urbana. Este grupo tiene algunas características similares al clúster 4, de barrios periurbanos de sectores bajos con marcadas restricciones en infraestructura. Junto con formas irregulares de tenencia, la lejanía de estos barrios explica también parte de las privaciones en materia de infraestructura urbana, por lo que se agrega, por ejemplo, la carencia de agua de red. Si bien algunas limitaciones siguen estando presentes, los clústeres 2 y 3 muestran una mayor dotación y acceso a los servicios urbanos, por lo que en ambos casos hablamos de barrios suburbanos relativamente consolidados. Sin embargo, mientras que el 2 socialmente comprende a sectores bajos, el 3 se compone de barrios de sectores medios-bajos, ya que comparativamente tienen valores más altos en términos de vivienda, educación y poder adquisitivo. 


\section{TABLA 2. MEDIAS DE LOS CLÚSTERES EN CADA DIMENSIÓN DE ANÁLISIS}

\begin{tabular}{|c|c|c|c|c|c|c|c|c|c|c|}
\hline \multirow[b]{2}{*}{ Clúster } & \multicolumn{6}{|c|}{ Variables socio-residenciales } & \multicolumn{4}{|c|}{ Régimen de tenencia } \\
\hline & $\begin{array}{l}\mathrm{NBI} \\
(\sin )\end{array}$ & $\begin{array}{l}\text { Red de } \\
\text { agua }\end{array}$ & Cloacas & $\begin{array}{l}\text { Gas de } \\
\text { red }\end{array}$ & Vivienda & Bienes & Educación & Propietario & Inquilino & $\begin{array}{l}\text { Otra } \\
\text { tenencia }\end{array}$ \\
\hline 1 & $-3,52$ & 0,26 & $-0,79$ & $-2,62$ & $-2,97$ & $-3,05$ & $-1,64$ & 0,36 & $-1,36$ & 1,13 \\
\hline 2 & $-1,46$ & 0,45 & 0,41 & $-0,33$ & $-1,78$ & $-1,24$ & $-1,42$ & 0,36 & $-1,07$ & 0,81 \\
\hline 3 & $-0,52$ & 0,43 & $-1,09$ & $-0,24$ & $-0,65$ & $-0,50$ & $-0,89$ & 0,41 & $-0,65$ & 0,27 \\
\hline 4 & $-0,64$ & $-0,75$ & $-1,02$ & $-2,47$ & $-1,15$ & $-1,48$ & $-0,75$ & $-0,21$ & $-0,91$ & 1,28 \\
\hline 5 & $-0,53$ & 0,47 & 0,77 & 0,17 & $-0,17$ & $-0,30$ & $-0,61$ & $-0,66$ & 0,23 & 0,49 \\
\hline 6 & 0,47 & 0,48 & 1,02 & 0,61 & 0,49 & 0,38 & 0,54 & $-1,25$ & 1,70 & $-0,50$ \\
\hline 7 & 0,80 & 0,25 & 0,66 & 0,44 & 0,91 & 1,19 & 1,26 & $-4,34$ & 0,43 & 4,49 \\
\hline 8 & 0,45 & $-0,15$ & $-1,01$ & 0,18 & 0,38 & 0,56 & 0,75 & 0,39 & $-0,14$ & $-0,29$ \\
\hline 9 & 0,75 & 0,28 & $-0,19$ & 0,64 & 0,85 & 1,15 & 1,31 & 0,08 & 0,77 & $-0,97$ \\
\hline 10 & 0,53 & 0,47 & 1,07 & 0,66 & 0,48 & 0,20 & $-0,76$ & 1,10 & $-0,84$ & $-0,30$ \\
\hline 11 & 0,29 & $-2,85$ & $-1,27$ & $-0,99$ & 0,20 & $-0,12$ & 0,64 & 0,10 & $-0,27$ & 0,19 \\
\hline
\end{tabular}

Fuente: elaboración propia. 


\section{BarRIOS CÉNTRICOS CONSOLIDADOS: 5 Y 6}

Se localizan en zonas céntricas, muy consolidadas en materia de infraestructura y servicios urbanos. No obstante, el clúster 6 abarca a unidades espaciales en el núcleo del centro urbano y que muestran un elevado nivel socioeconómico, por lo que hablamos de barrios micro-céntricos consolidados de sectores medios-altos y altos. Además, en consonancia a los altos valores del suelo que lo distinguen, allí se observan los mayores porcentajes de inquilinato de toda la ciudad (como contracara, una menor proporción de propietarios). En cambio, el clúster 5 abarca a barrios macro-céntricos consolidados de sectores medios y medios-bajos, que se ubican en un primer anillo de expansión.

\section{Barrios Suburbanos de Sectores medios-altos: 8 y 9}

En parte por la búsqueda del paisaje, la ciudad de Bariloche ha crecido en extensión a lo largo del Lago Nahuel Huapi hacia el Oeste y más recientemente al Este. Aquí se localizan las clases mediasaltas, en barrios con una dotación razonablemente buena de infraestructura y servicios urbanos, con la excepción particular del acceso a la red de cloacas, que se ha visto restringida por la distancia al centro o la menor antigüedad de algunos de estos barrios. La existencia de algunas restricciones en materia de infraestructura se verifica especialmente en el clúster 8, de barrios suburbanos relativamente consolidados de sectores medios-altos. En cambio, estas limitaciones son mucho más aisladas en los barrios suburbanos consolidados de sectores mediosaltos y altos del clúster 9, en parte por su mayor cercanía al centro. Vale destacar que allí se observa una elevada proporción de inquilinos, solo por detrás del micro-centro (6).

\section{BarRios de ViVIENDAS E INFRAESTRUCtURA SOCIAL PARA SECTORES MEDIOS-BAJOS (ENTRE EL CENTRO Y LOS SUBURBIOS): 10}

Aquí viven sectores medios-bajos y bajos, pero cuyas condiciones de infraestructura urbana son buenas (prácticamente, iguales al clúster 6) y la proporción de propietarios es la más alta de la ciudad. Estas características se explican, en buena medida, por el hecho de tratarse de viviendas sociales construidas por el Estado, en muchos casos hace varias décadas y en los márgenes del macrocentro o en zonas suburbanas cercanas, dada la mayor disponibilidad de tierras en ese momento. Si bien presentan problemas de mantenimiento y conflictos al interior de sus comunidades, son barrios que, desde un punto de vista meramente físico y de accesibilidad, se encuentran completamente integrados a la ciudad. 


\section{FIGURA 2. MAPA DE BARILOCHE CON LOS TIPOS DE BARRIOS.}

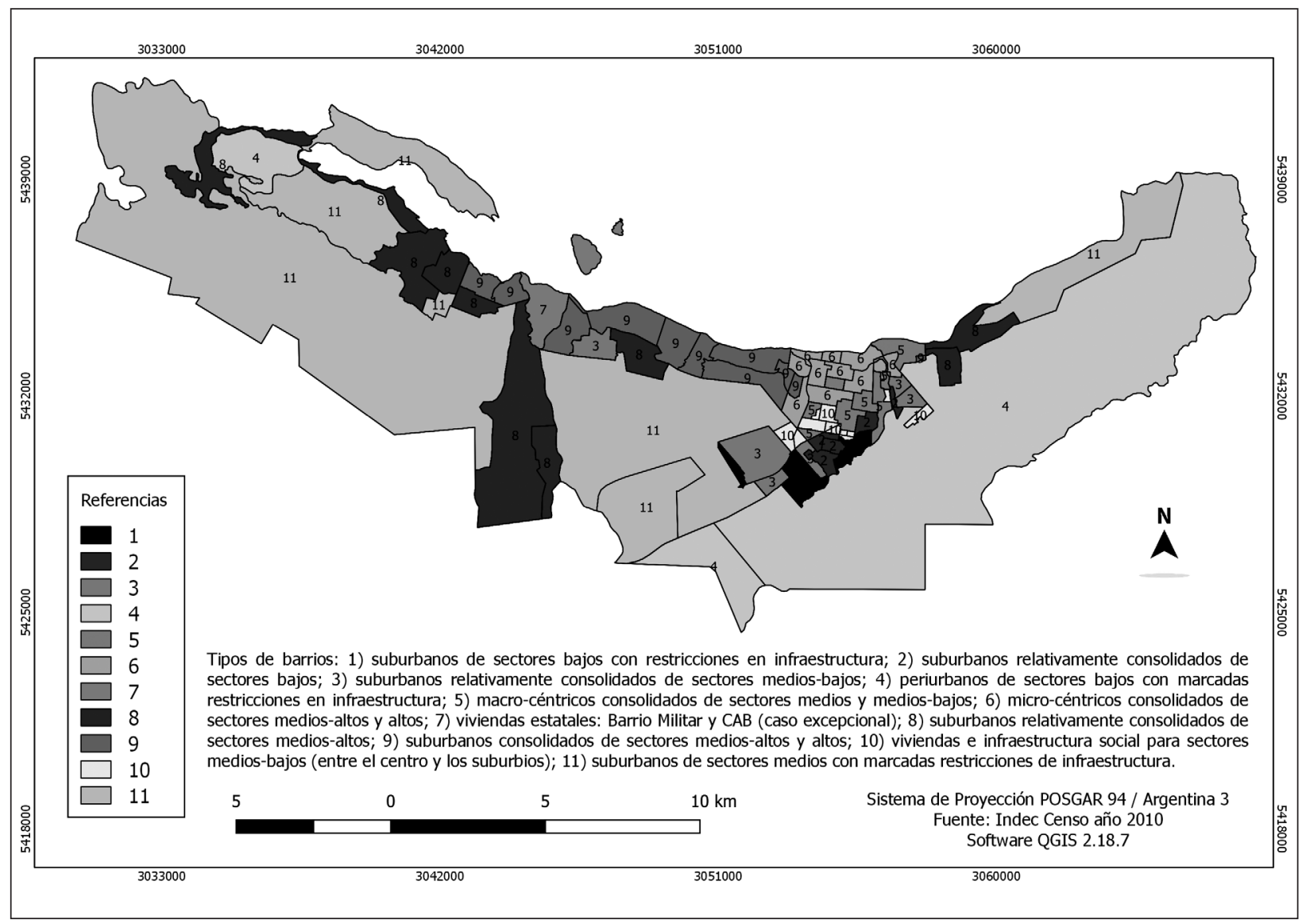

Fuente: elaboración propia en base datos del INDEC. 


\section{Barrios SUbuRbanos de SECTORES MEdIOS CON MARCADAS RESTRICCIONES DE INFRAESTRUCTURA: 11}

Se trata de barrios con menor densidad de población (por eso el gran tamaño de las unidades censales) donde viven sectores medios y algunos más acomodados pero que, por preferencias en su localización, priorizan el entorno natural, la cercanía con el bosque y/o el lago, en espacios alejados del centro, con algunas barreras físicas (cerros, lagos, lagunas) y donde la dotación de infraestructura y servicios urbanos es todavía muy deficitaria. En algunos casos, la combinación de lejanía y menor consolidación de infraestructura se traduce además en un menor costo del suelo, lo que facilita el acceso de estas clases sociales.

\section{TIPOS DE BARRIOS, SRS E INSERCIÓN LABORAL EN BARILOCHE}

En línea con la definición de SRS adoptada, el ejercicio anterior nos permitió identificar distintos conglomerados de barrios de relativa homogeneidad sociorresidencial hacia adentro, pero heterogéneos entre sí, como así también apreciar las zonas de la ciudad donde estos grupos tienden a localizarse. Adentrándonos ya en la cuestión de la inserción laboral, el análisis de la varianza (Tabla 3) nos muestra, en primera instancia, que las tasas de actividad, empleo y desempleo (al momento del censo 2010) son significativamente diferentes entre los distintos clústeres, lo cual vuelve a dar cuenta del grado de diferenciación barrial en Bariloche.

Por un momento, conviene enfocarnos en los barrios de clases medias-bajas y bajas de Bariloche, ya que allí se observan las peores realidades socioeconómicas de la ciudad. Nos referimos explícitamente a los barrios que componen los clústeres 1 a 4 , pero también el 5 y el 10, donde el aspecto compartido que refleja la SRS de estos barrios es, por sobre todo, el muy bajo nivel educativo del jefe de hogar (Tabla 2), una dimensión que en varios trabajos ha sido considerada como uno de los mejores predictores del grado de segregación socioeconómica de los hogares (Kaztman y Retamoso, 2005; Molinatti, 2013a; Perelman, 2011). Al margen de esto, hemos visto que es posible trazar algunas diferencias en términos residenciales. Mientras que los clústeres 5 y 10 se ubican en áreas macro-céntricas (o en los márgenes del centro) con infraestructuras urbanas consolidadas y buenas condiciones habitacionales, los barrios periféricos (clústeres 1 a 4) presentan diferentes clases de privaciones respecto a la calidad habitacional y/o al acceso a servicios urbanos.

Ahora bien, la pregunta que nos hacemos puntualmente en este apartado es si estos barrios socioeconómicamente segregados comparten o no algún patrón similar en términos de la inserción laboral 
TABLA 3. ANÁLISIS DE LA VARIANZA (ANOVA)

\begin{tabular}{lllll} 
& $\begin{array}{l}\text { Suma de } \\
\text { cuadrados }\end{array}$ & $\begin{array}{l}\text { Grados de } \\
\text { libertad }\end{array}$ & $\begin{array}{l}\text { Media } \\
\text { cuadrática }\end{array}$ & F \\
\hline T. Actividad & 26,335 & 10 & 2,633 & $3,500^{* *}$ \\
\hline T. Empleo & 40,990 & 10 & 4,099 & $7,727^{* * *}$ \\
T. Desempleo & 38,244 & 10 & 3,824 & $6,685^{* * *}$
\end{tabular}

Fuente: elaboración propia.

Nivel de significación: ${ }^{*} \mathrm{p}<0,05 ;{ }^{*} \mathrm{p}<0,01 ; * * * \mathrm{p}<0,001$.

de la población que allí reside y, en todo caso, si este escenario difiere a su vez del resto de los vecindarios de la ciudad. En particular, uno de los problemas más estudiados en su relación con la SRS ha sido el desempleo (Cunha y Jakob, 2010; Dujardin et ál., 2008; Kaztman y Retamoso, 2005; Perelman, 2011; Sabatini et ál., 2001; Sabatini y Wormald, 2013). En la Figura 3 podemos apreciar la tasa de desempleo promedio para los distintos clústeres, expresada nuevamente como $Z$ score para mantener la línea expositiva anterior y hacer incluso más visuales las diferencias. Como se puede observar allí, los conglomerados de barrios que aglutinan a los hogares de peores condiciones socioeconómicas efectivamente presentan las tasas de desocupación más altas de toda la ciudad (en todos los casos, por encima de la media general). Por lejos, las tasas de desempleo más preocupantes se manifiestan en el clúster 1 , seguido por el 2 . Otro aspecto llamativo es que la desocupación es más alta en los barrios de viviendas sociales (10) y el área macro-céntrica (5) que en los barrios suburbanos más alejados del 3 y 4.

Esto podría tomarse al menos como evidencia preliminar en contra de la tesis del desajuste espacial en el caso de Bariloche, si tenemos en cuenta que gran parte de los puestos de trabajo de la ciudad se desarrollan en el centro o sus alrededores. Por otro lado, lo relativo al clúster 10 va en línea con los resultados cosechados por Sabatini y Wormald (2013) al estudiar la segregación de la vivienda social en Chile, aunque en este caso son conjuntos de viviendas de localización periférica y no macro-céntrica como en Bariloche. Cabe entonces en este punto preguntarse, aunque no estamos en condiciones de responder ciertamente, por qué la 
desocupación es más elevada en barrios de viviendas sociales, a pesar de no estar localizados en las periferias más alejadas del centro.

La contracara del panorama anterior se da en los barrios de clases medias y medias-altas, tanto del micro-centro (6) como de áreas suburbanas (8, 9 y 11), con tasas de desempleo considerablemente por debajo del promedio general y relativamente parejas entre sí. El caso más sobresaliente es el 11, que presenta las menores tasas de desocupación de toda la ciudad a pesar de no ser los barrios con el status socioeconómico más elevado. A modo de especulación, lo que podría estar por detrás de estos valores es la mayor presencia de clases medias trabajadoras (con un cierto nivel educativo y quizás más jóvenes), en el sentido de que obtendrían principalmente sus ingresos del empleo (asalariado), mientras que en los otros barrios de clases medias-altas y altas podrían haber fuentes alternativas de recursos que se complementen con la inserción laboral, o en todo caso, un mix de hogares con ingresos de diferentes fuentes (varios de ellos no atados al empleo asalariado). Algo de esto puede verse reflejado también en la Figura 4, ya que son nuevamente los barrios del clúster 11 los que muestran las tasas más altas de actividad y empleo en Bariloche, una situación bien diferente a la que se observa en el micro-centro (6), donde las tasas están en torno a la media general (actividad) o levemente por encima de la misma (empleo). Como es usual también en otras ciudades, una buena parte de la población del centro suele tener edades avanzadas y estar ya en la inactividad (jubilados y pensionados).

En el otro extremo, las tasas más bajas de empleo y actividad vuelven a observarse en los clústeres 1 y 2, lo cual da cuenta no solo de la baja empleabilidad de la población que habita en estos barrios segregados, sino también del mayor peso de la inactividad en estos vecindarios. Esto último puede deberse a diferentes razones, como modelos disfuncionales (los ni-ni entre los jóvenes, la incidencia de planes de asistencia social entre los adultos), inserciones laborales precarias, esporádicas u ocasionales (las changas y la economía popular), roles de género bien preestablecidos en el hogar (las mujeres al cuidado de los niños y ancianos), el desaliento en la búsqueda de empleo, entre otras. Obsérvese incluso que el posible caso de los desocupados desalentados, que técnicamente se contabilizan dentro de los inactivos, podría llevar a ampliar las negativas cifras de desempleo que se evidencian en estos barrios, si acaso estos miembros del hogar volvieran a insertarse en el mercado de trabajo y buscaran un empleo. En otros términos, e independientemente del ejemplo del trabajador desalentado, si las tasas de actividad de estos vecindarios fueran más altas probablemente también lo serían las de desempleo, ya que hay más chances de que la población transite de la inactividad a la desocupación, al menos por un tiempo, de que se concrete un salto directo al 


\section{FIGURA 3. TASA DE DESEMPLEO (2010), SEGÚN CLÚSTER.}

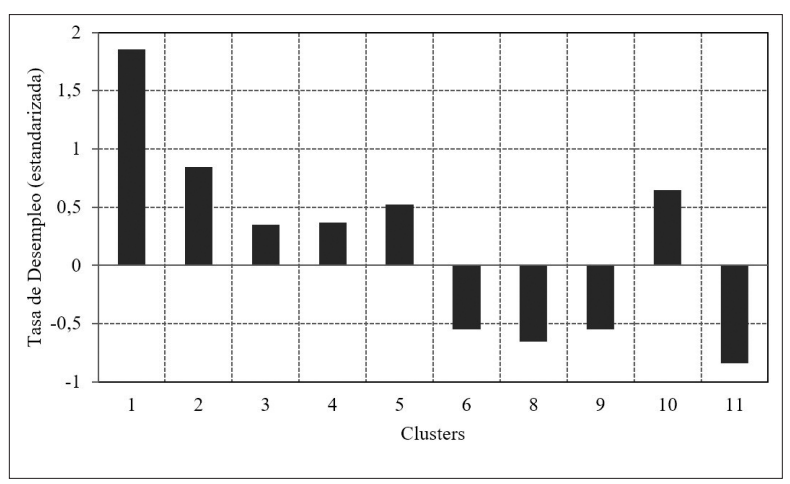

Fuente: elaboración propia.

empleo. En definitiva, estamos hablando de áreas de relegación donde se concentra el fenómeno de la marginalidad avanzada.

$\mathrm{Al}$ igual que antes, los clústeres 5 y 10 también se encuentran en una situación desfavorable, lo cual en el último caso vuelve a alinearse con Sabatini y Wormald (2013). Lo más interesante tal vez es la situación de los otros barrios suburbanos de clases medias-bajas y bajas ( 3 y 4) que, a diferencia del resto, muestran tasas de actividad cercanas a la media de toda la ciudad y muy similares a las del micro-centro (6), como así también tasas de empleo apenas por debajo del promedio. Siguiendo
FIGURA 4. TASAS DE ACTIVIDAD Y EMPLEO (2010), SEGÚN CLÚSTER.

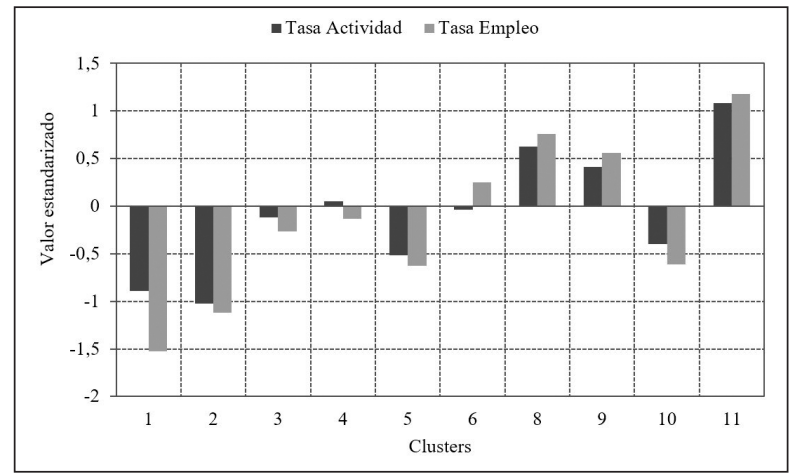

Fuente: elaboración propia.

con las mismas líneas argumentativas, es posible suponer que estamos frente aquí a clases mediasbajas trabajadoras, que no sufrieron tanto los procesos de desproletarización que afectaron a otros segmentos sociales.

Todas las reflexiones anteriores se mantienen si en lugar de haber tomado los valores estandarizados hubiéramos considerado las diferencias absolutas con respecto a la media (en puntos porcentuales) para cada una de las tasas (Tabla 4). Como puede apreciarse, además, la magnitud de estas distancias es considerable en muchos casos. 
TABLA 4. DIFERENCIAS EN PUNTOS PORCENTUALES RESPECTO A LA MEDIA GENERAL

\begin{tabular}{|lccc|}
\hline Clúster & T. Desempleo & T. Actividad & T. Empleo \\
\hline 1 & $\mathbf{6 , 6}$ & $\mathbf{- 3 , 3}$ & $-\mathbf{7 , 7}$ \\
\hline 2 & $\mathbf{3 , 0}$ & $\mathbf{- 3 , 8}$ & $\mathbf{- 5 , 6}$ \\
\hline 3 & 1,3 & $-0,4$ & $-1,3$ \\
\hline 4 & 1,3 & 0,2 & $-0,7$ \\
\hline 5 & 1,8 & $-1,9$ & $-\mathbf{3 , 1}$ \\
\hline 6 & $-2,0$ & $-0,1$ & 1,2 \\
\hline 8 & $-2,3$ & 2,3 & $\mathbf{3 , 8}$ \\
\hline 9 & $-1,9$ & 1,5 & 2,8 \\
\hline 10 & 2,3 & $-1,5$ & $\mathbf{- 3 , 1}$ \\
\hline 11 & $-\mathbf{3 , 0}$ & $\mathbf{4 , 0}$ & $\mathbf{5 , 9}$ \\
\hline
\end{tabular}

Fuente: elaboración propia. 


\section{Conclusiones}

La segregación residencial socioeconómica puede considerarse como uno de los principales obstáculos hacia el objetivo de integración urbana previsto en la Nueva Agenda Urbana de Hábitat III, en la medida que aparece asociada a diferentes manifestaciones de la desintegración y exclusión social, y suele actuar, a su vez, como un mecanismo de reproducción de las desigualdades socioeconómicas. En particular, a lo largo de esta investigación buscamos realizar un aporte a la discusión sobre los vínculos entre la SRS y la inserción laboral de la población, una cuestión clave en América Latina, ya que tiende a condicionar o determinar su nivel de bienestar. Para ello, mediante una combinación de técnicas de análisis multivariado desarrollamos una tipología (y diferenciación) barrial, que luego contrastamos con el tipo de integración en el mercado de trabajo de la población que allí reside. Vale destacar que, a diferencia de una parte de la literatura (por ejemplo, de efecto vecindario) que busca analizar efectos causales desde las características barriales hacia la inserción laboral de los individuos, aquí propusimos un abordaje más prudente, que considera la posibilidad de causalidades de doble vía o circulares, para evitar caer así en determinismos espaciales.

El análisis exploratorio de la inserción laboral de la población según el tipo de barrio en que habita nos mostró que las tasas de actividad, empleo y desempleo (en 2010) eran significativamente diferentes entre los distintos clústeres, lo cual es otra manifestación del grado de diferenciación barrial existente en Bariloche. En línea con la evidencia previa de ciudades latinoamericanas, los barrios que muestran los peores indicadores de actividad, empleo y desempleo son los barrios populares, de sectores medios-bajos o bajos, ya sea suburbanos (principalmente 1 y 2 y, en menor medida, 3 y 4), macro-céntricos (5) o los complejos de viviendas sociales (10) en un espacio de transición entre los anteriores. Pero además de las diferencias en la localización, mientras que los clústeres 5 y 10 presentan un elevado nivel de consolidación urbana, los clústeres 1 a 4 exhiben diferentes restricciones en el acceso a infraestructura.

Parte de la evidencia empírica que presentamos podría contradecir a la tesis del desajuste espacial, ya que barrios relativamente bien integrados a la trama urbana por su cercanía relativa al centro de la ciudad (donde se desarrollan el grueso de las actividades económicas), como los clústeres 5 y 10, presentan niveles de desempleo superiores que los barrios suburbanos populares del 3 y 4 . Particular interés genera el clúster 10, dado su origen producto de programas de vivienda social. De esta forma, se puede reflexionar sobre la capacidad del Estado de incidir en la integración urbana plena de la población si solo se limita al acceso a la vivienda y servicios básicos. La evidencia que presentamos parece dar cuenta de un sector 
de la población con demandas habitacionales relativamente satisfechas, incluyendo una relativamente buena ubicación y conectividad, pero con déficit de integración urbana en cuanto a su inserción sociolaboral. Al contrario, barrios suburbanos con peor accesibilidad y mayores carencias habitacionales y de infraestructura, como los clústeres 3 y 4 , aparecen como más dinámicos en materia de integración al mercado de trabajo y, con ello, a las posibilidades de progreso social.

En un sentido similar, el clúster 11, de localización suburbana-periférica y bajo nivel de consolidación urbana (incluyendo además una muy mala accesibilidad al transporte público), presenta las menores tasas de desempleo de la ciudad (y las mayores de actividad y empleo), a pesar de no ser estrictamente los barrios de más alto nivel socioeconómico, lo que podría ser compatible con una mayor presencia de clases medias trabajadoras. En cambio, los altos niveles de desempleo que se observan en los clústeres 1 y 2 (también de inserción urbana periférica y con limitado acceso a infraestructura), sí podrían abonar las ideas detrás del efecto vecindario y el desajuste espacial, mientras que las bajas tasas de empleo y actividad pueden reflejar procesos de desproletarización y marginalidad avanzada.
En este punto, podría pensarse que los bajos niveles de consolidación de la trama urbana y la ausencia o déficit en la provisión de ciertos servicios públicos en estos clústeres se relacionan en parte con los procesos más recientes de expansión de la mancha urbana, por tratarse de áreas periféricas de la ciudad. Esto se debe tanto a la generación de nuevos loteos y urbanizaciones para sectores medios, que pueden rastrearse en el clúster 11 , como así también a procesos de ocupación de suelo urbano ocioso por parte de grupos pertenecientes a los sectores populares, que de otra manera no tendrían posibilidades de acceder a la tierra y a la vivienda (por ejemplo, en el clúster 1).

Si bien los resultados están lejos de ser concluyentes y se pone de manifiesto la posibilidad a futuro de investigar estos vínculos más a fondo, esperamos que la evidencia exploratoria presentada pueda contribuir a complejizar la mirada un tanto lineal y determinista que aparece en la literatura al conectar las características barriales y la inserción laboral de la población, como así también a pensar un paquete de políticas públicas más diversificado para poder hacer frente a las distintas problemáticas que obstruyen la integración urbana. 


\section{Referencias bibliográficas}

Aguilar, A. G. y Mateos, P. (2011). Diferenciación sociodemográfica del espacio urbano de la Ciudad de México. EURE, 37(110), 5-30. doi:10.4067/ S0250-71612011000100001

Arriagada Luco, C. (2010). Segregación residencial según dos modelos de urbanización y bienestar: estudio comparado de las áreas metropolitanas del Gran Santiago, Toronto y Vancouver. Notas de Población, (91), 201-226.

Bosch, J. L., Suárez, S., y Olivares, G. (2004). La importancia de la generación de empleo como dinamizadora del desarrollo local en un centro turístico. Caso: San Carlos de Bariloche. Aportes y Transferencias, $8(2), 25-44$.

Brain, I., Cubillos, G., y Sabatini, F. (2007). Integración social urbana en la nueva política habitacional. Santiago: Pontificia Universidad Católica de Chile.

Cunha, J. M. P. y Jakob, A. A. E. (2010). Segregação socioespacial e inserção no mercado de trabalho na Região Metropolitana de Campinas. Revista Brasileira de Estudos de População, 27(1), 115-139. doi:10.1590/S0102-30982010000100008

Dujardin, C., Selod, H., y Thomas, I. (2008). Residential segregation and unemployment: the case of Brussels. Urban Studies, 45(1), 89-113. doi:10.1177\%2F0042098007085103
Grande, P. d. (2016). El formato Redatam. Estudios Demográficos y Urbanos, 31(3), 811-832. doi: 10.24201/ edu.v31i3.15

Groisman, F. y Suárez, A. L. (2010). Segregación residencial e inserción laboral en el Conurbano Bonaerense. Población de Buenos Aires, 7(11), 7-28.

Hair, J., Black, W., Babin, B., y Anderson, R. (2010). Multivariate data analysis. Londres: Pearson.

Johnson, R. y Wichern, D. (2008). Applied multivariate statistical analysis. Londres: Pearson.

Kaztman, R. (1999). Activos y estructuras de oportunidades de estudios sobre las raíces de la vulnerabilidad social en Uruguay. Montevideo: CEPAL-PNUD.

Kaztman, R. y Retamoso, A. (2005). Segregación espacial, empleo y pobreza en Montevideo. Revista de la CEPAL, (85), 131-148.

Korsu, E. y Wenglenski, S. (2010). Job accessibility, residential segregation and risk of long-term unemployment in the Paris region. Urban Studies, 47(11), 2279-2324. doi:10.1177/0042098009357962

Marcos, M., Mera, G., y Di Virgilio, M. M. (2015). Contextos urbanos de la ciudad de Buenos Aires: una propuesta de clasificación de la ciudad según tipos de hábitat. Papeles de Población, 21(84), 161-196.

Matossian, B. (2015). División social del espacio residencial y migraciones: el caso de San Carlos de Bariloche, Argentina. EURE, 41(124), 163-184. doi:10.4067/S0250-71612015000400008 
Mikelbank, B. A. (2011). Neighborhood déjà vu: Classification in metropolitan Cleveland, 1970-2000. Urban Geography, 32(3), 317-333. doi:10.2747/0272-3638.32.3.317

Molinatti, F. (2013a). Segregación residencial e inserción laboral en la ciudad de Córdoba. EURE, 39(117), 117-145. doi:10.4067/ S0250-71612013000200006

Molinatti, F. (2013b). Segregación residencial socioeconómica en la ciudad de Córdoba (Argentina): Tendencias y patrones espaciales. Revista Invi, 28(79), 61-94. doi:10.4067/S0718-83582013000300003

Niembro, A. (2011). El desarrollo (pendiente) de Bariloche. Reflexiones a cien años de la Comisión de Estudios Hidrológicos. Revista Pilquen, Sección Ciencias Sociales, 14(1), 1-17.

Niembro, A., Guevara, T., y Cavanagh, E. (2019). Segregación urbana e infraestructura en América Latina: una tipología de los barrios de Bariloche. Bariloche, Argentina: Centro Interdisciplinario de Estudios sobre Territorio, Economía y Sociedad (CIETES), Universidad Nacional de Río Negro.

Owens, A. (2012). Neighborhoods on the rise: A typology of neighborhoods experiencing socioeconomic ascent. City \& Community, 11(4), 345-369. doi:10.1111/j.1540-6040.2012.01412.x

Perelman, L. (2011). Diferencias socioespaciales e inserción laboral en la ciudad de Buenos Aires. Buenos Aires: CEDEM.

Rodríguez Merkel, G. (2013). El uso de zonas censales para medir la segregación residencial:
Contraindicaciones, propuesta metodológica y un estudio de caso: Argentina 19912001. EURE, 39(118), 97-122. doi:10.4067/ S0250-71612013000300005

Rodríguez Vignoli, J. (2008). Movilidad cotidiana, desigualdad social y segregación residencial en cuatro metrópolis de América Latina. EURE, 34(103), 49-71. doi: 10.4067/S0250-71612008000300003

Rodríguez Vignoli, J. y Arriagada Luco, C. (2004). Segregación residencial en la ciudad latinoamericana. EURE, 30(89), 5-24. doi:10.4067/ S0250-71612004008900001

Ruiz-Tagle, J. (2016). La segregación y la integración en la sociología urbana: revisión de enfoques y aproximaciones críticas para las políticas públicas. Revista INVI, 31(87), 9-57. doi:10.4067/ S0718-83582016000200001

Ruiz-Tagle, J. y López, E. (2014). El estudio de la segregación residencial en Santiago de Chile: revisión crítica de algunos problemas metodológicos y conceptuales. EURE, 40(119), 25-48. doi:10.4067/ S0250-71612014000100002

Sabatini, F., Cáceres, G., y Cerda, J. (2001). Segregación residencial en las principales ciudades chilenas: Tendencias de las tres últimas décadas y posibles cursos de acción. EURE, 27(82), 21-42. doi:10.4067/S0250-71612001008200002

Sabatini, F. y Wormald, G. (2013). Segregación de la vivienda social: reducción de oportunidades, pérdida de cohesión. En F. Sabatini, G. Wormald y A. Rasse (Eds.), Segregación de la vivienda social: 
ocho conjuntos en Santiago, Concepción y Talca. Santiago de Chile: Pontificia Universidad Católica de Chile.

Salvia, A. y Vergara, A. (2011). Marginalidad, desempleo y segregación residencial en la Argentina en un contexto de crecimiento (2006-2008). En A. Salvia (Ed.), Deudas sociales en la Argentina posreformas. Buenos Aires: Biblos.

Sánchez, D., Sassone, S., y Matossian, B. (2007). Barrios y áreas sociales de San Carlos de Bariloche: análisis geográfico de una ciudad fragmentada. IX Jornadas Argentinas de Estudios de Población. Huerta Grande, Córdoba: Asociación de Estudios de Población de la Argentina.

Slater, T. (2013). Your life chances affect where you live: A critique of the 'cottage industry' of neighbourhood effects research. International Journal of Urban and Regional Research, 37(2), 367-387. doi:10.1111/j.1468-2427.2013.01215.x

Telles, E. E. (1995). Structural sources of socioeconomic segregation in Brazilian metropolitan areas. American Journal of Sociology, 100(5), 1199-1223.

Vicino, T. J., Hanlon, B., y Short, J. R. (2011). A typology of urban immigrant neighborhoods. Urban Geography, 32(3), 383-405. doi:10.2747/0272-3638.32.3.383 